\title{
Bioactive Compounds and Essential Oils as Acetylcholinesterase Inhibitors
}

\author{
Edna Kurgat ${ }^{1}$, Doaa Ghareeb ${ }^{2,}$ * \\ ${ }^{1}$ Department Public Health, South Eastern Kenya University, Nairobi, Kenya \\ ${ }^{2}$ Department Biochemistry, Faculty of Science, University of Alexandria, Alexandria, Egypt \\ Email address: \\ ednakurgat83@gmail.com (E. Kurgat), ghareeb $a$ gmail.com (D. Ghareeb) \\ ${ }^{*}$ Corresponding author
}

\section{To cite this article:}

Edna Kurgat, Doaa Ghareeb. Bioactive Compounds and Essential Oils as Acetylcholinesterase Inhibitors. American Journal of Biomedical and Life Sciences. Vol. 7, No. 6, 2019, pp. 155-158. doi: 10.11648/j.ajbls.20190706.15

Received: September 20, 2019; Accepted: November 6, 2019; Published: December 2, 2019

\begin{abstract}
As total life expectancy increases, the prevalence of age-related diseases such as Alzheimer's is also increasing. Many hypotheses about Alzheimer's disease have been developed, including cholinergic neuron damage. Acetylcholine is a major neurotransmitter in the brain and cholinergic deficits leads to cognitive dysfunction and decline. Despite decades of research and advances in our understanding of its aetiology and pathogenesis, current pharmacotherapeutic options for $\mathrm{AD}$ are still very limited and represent an area of need that is currently unmet. In abnormal activation of AChE, acetylcholine will degrade rapidly, especially in the brain and this is associated with Alzheimer's disease (AD). It has been shown that theraphy with essential oils from medicinal plants can improve cognitive performance in Alzheimer's disease patients. Eugenol from these essential oils is reported to inhibit acetylcholinesterase, both in vitro and in vivo. This paper is set to Determine inhibitory/stimulatory effect of tested extracts on acetylcholine esterase (AChE) activity. The sampled out plant extracts include Thymus vulgaris, Berberis vulgaris and Calluna vulgaris with which inhibition or activation by different chemical catalysts is performed to establish their effects in the tested natural extracts. Experimental design is used where the reagents are determined and chemical reactions performed in the procedures as outlined in the methodology section. The results of the cholinergic/ anticholinergic effect of tested natural extracts are then recorded. This study reflects that most of the extracts inhibited AChE activity with berberis vulgaris showing highest inhibitory effect.
\end{abstract}

Keywords: Alzheimer's Disease (AD), Acetylcholine (ACh), Acetylcholinesterase (AChE, Essential Oils (Eos)

\section{Introduction}

Inhibition of Cholinesterases, mainly Acetylcholinesterase (AChE) and therefore prevention of acetylcholine degradation in synapses of cholinergic system is one of the most accepted palliative therapy opportunities for Alzheimer's disease (AD) today. Thus, it is no surprise that there is a recent increased interest in the use of essential oils, as alternative medicine. Essential oils can significantly reduce agitated behavior in $\mathrm{AD}$ patients (Ayaz et al., 2017) [1]. Since the introduction of the first cholinesterase inhibitor in 1997, most clinicians would consider the cholinergic drugs, donepezil, rivastigmine and galantamine to be the first line pharmacotherapy for mild and moderate AD. These compounds have been reported to have their adverse effects including gastrointestinal disturbances and problems associated with bioavailability, high cost, and short half-life [2] which necessitates the interest in finding better AChE inhibitors from natural resources.

Desirable properties of botanical extracts or natural product based compounds include a comparatively better penetration of the blood-brain barrier better than the pharmaceutical options and better specificity for human type AChE. Although the anticholinesterase activity of many plant compounds have been demonstrated, in vitro laboratory trials using essential oil extracts of Thymus vulgaris and Berberis vulgaris plants are very limited. Plant-derived essential oils exhibit pharmacological properties traceable to the presence of various structurally diverse bioactive chemical 
components and are increasingly harnessed for their anticholinesterase (AChE) properties. Recent studies on the acetyl-cholinesterase inhibiting potentials of commercial essential oils documented Eugenol as predominant bioactive compounds [3]. Considering the importance of sufficient knowledge base for accurate recommendations on the use of plant extracts, this study found the main functional component alkaloids such as which is the main active ingredient of Berberis vulgaris.

\section{Material and Methods}

\subsection{Bioactive Compounds and Essential Oils as Acetylcholinesterase Inhibitors}

Several reviews on the newly discovered AChE inhibitors obtained from plants have also been published over the last years [4]. The majority of these AChE inhibitors belong to the alkaloid group, including indole, isoquinoline, quinolizidine, piperidine and steroidal alkaloids. On the other hand, several non-alkaloidal and potent AChE inhibitors have been obtained from natural sources, including terpenoids, flavonoids, and EO. Interestingly, although literature demonstrates to be rich in the study on AChE inhibitors obtained from plants, this issue keeps on being the centre of attention for research as confirmed by the increasing number of studies published every year.

Methanolic extracts of Berberis vulgaris Thymus vulgaris medicinal herbs for improvement of memory and cognition in old age have been tested for cholinesterase inhibitory properties and significant inhibition of the enzyme was shown by extracts [3]. It has been suggested that, the main functional components found in Berberis extract are alkaloids such as berberine which is the main active ingredient of Berberis vulgaris [3], therefore the compound might have a therapeutic potential for treatment of Alzheimer's disease. Studies concerning the AChE inhibitory activity and chemical composition of commercial essential oils of Thymus vulgaris performed by demonstrated for the first time that eugenol was a potent AChE inhibitor.

\subsubsection{Pharmacological Activities}

Berberis vulgaris has demonstrated wide range of pharmacological activities including antihypertensive, antiinflammatory, antioxidant, antidepressant, anticancer, antidiarrhoeal, cholagouge, hepatoprotective and above all antimicrobial. Recent findings show that the extract of Berberidacea prevents neurological damage due to oxidative stress and it might act as a novel cholesterollowering compound [5]. The main functional component found in Berberis compound have a therapeutic potential for treatment of Alzheimer's disease [3]. Many EOs and their monoterpenes have been also investigated for their capacity of inhibiting AChE. For example, studies concerning the AChE inhibitory activity and chemical composition of commercial essential oils performed by (Dohi et al, 2009) demonstrated for the first time that eugenol was a potent AChE inhibitor. Recently, a study concerning the AChE inhibitory of Thymus vulgaris EOs was reported [6].

\subsubsection{Photochemistry}

Phytochemicals are natural bioactive compound widely distributed in plants, animals, microbes, and other forms of life. The principal examples of natural products including alkaloids, terpenoids, steroids, polyphenols, and flavonoids have rational uses and are found in varying amounts in different species. Their presence in the food chains has significant role which is to work with nutrients and dietary fibre to protect against disease [12].

The main compounds, found in various species of Berberis are berberine and berbamine. Phytochemical analysis of the crude extract of $B$. vulgaris has revealed the presence of alkaloids, tannins and phenolic compounds [13] in addition recent study showed triterpenes and monoterpenes isolated from ethanolic extract. The alkaloids: berberamine, berberine [14], has also been extracted from ethyl acetate extracted also from $B$. vulgaris [15].

\subsection{Liver Homogenate Preparation}

One gram of liver was homogenized with 9 volume of phosphate buffer, $0.1 \mathrm{M}, \mathrm{pH} 7.4$ and then centrifuged at 3000 r.p.m for $15 \mathrm{~min}$ and the sap was saved to be used as liver homogenate.

Determination of inhibitory/stimulatory effect of tested extracts on acetylcholine esterase (AChE) activity.

1. Principal

Acetyl cholinesterase (AChE) catalyzes the hydrolysis of acetylthiocholine (ATCh) upon hydrolysis, this substrate analog produces into acetate and thiocholine. Thiocholine, in the presence of the highly reactive dithiobisnitro-benzoate (DTNB) ion generates a yellow color, which is visible and can be quantitatively monitored by spectrophotometric absorption at $405 \mathrm{~nm}$.

2. Reagents

a. Dithiobis 2-nitrobenzoic acid (DTNB)

b. Phosphate buffer (0.1M pH 7.4)

c. Acetylthiocholin iodide (ACTI)-substrate

3. Procedure

AChE activity was measured according to the method of Ell man [8] whereby $150 \mu \mathrm{l}$ of phosphate buffer $(0.1 \mathrm{M}, \mathrm{pH} 8)$ were added to a mixture of $60 \mu \mathrm{l}$ of liver homogenate and $20 \mu \mathrm{l}$ of the extracts (test), or organic solvent (control) then incubated for $45 \mathrm{~min}$ at $37^{\circ} \mathrm{C}$. Five micro liter of substrate ACTI $(75 \mathrm{mM})$ were added, mixed well and incubated for $15 \mathrm{~min}$ at $37^{\circ} \mathrm{C}, 60 \mu \mathrm{l}$ of DTNB $(0.32 \mathrm{mM})$ were added and left for $5 \mathrm{~min}$ to stop the reaction. The absorbance was measured against the blank at $405 \mathrm{~nm}$ and specific AChE activity was calculated as follows:

The values obtained were analyzed; blank reading was subtracted from sample readings.

Specific enzyme activity $=[\mathrm{A}] \mathrm{x}$ [Total volume in cuvette in $\mu \mathrm{L}$ ] / [Molar extinction coefficient of DTNB] $\mathrm{x}$ [Volume 
of liver homogenate in $\mu \mathrm{L}$ ] $\mathrm{x}$ [Protein concentration in $\mathrm{mg}$ / $\mathrm{mL}]$

Where, specific activity $=$ moles of substrate hydrolyzed $/$ min $/ \mathrm{mg}$ of protein $\mathrm{A}=$ absorbance Molar extinction of $\mathrm{DTNB}=1.36 \times 104$

\section{Results}

\subsection{The Cholinergic / Anti-cholinergic Effect of Tested Natural Extracts}

Most of the tested fractions extracts showed potent inhibitory effect toward AChE except PEG and 95\% ethanol fractions of Thymus vulgaris that showed stimulatory effect by $19 \%$ and $11.5 \%$ respectively at $\mathrm{p}<0.05$. The highest inhibitory effect was shown in PEG fraction of Berberis vulgaris by $32 \%$ whereas water fraction of Calluna vulgaris showed the lowest effect by $2 \%$ at $p<0.05$.

Table 1. Percentage Inhibition or Activation on Acetylcholine Esterase $(A C h E)$ in the Presence of Tested Natural Fractions.

\begin{tabular}{lll}
\hline Extract & Solvent & \% activation / inhibition \pm SD \\
\hline \multirow{4}{*}{ Thymus vulgaris } & Water & $-13.20 \pm 4.79$ \\
& PEG & $+19.00 \pm 3.90$ \\
& 20\% ethanol & $-12.84 \pm 5.00$ \\
& $95 \%$ ethanol & $+11.50 \pm 4.80$ \\
& Water & $-16.60 \pm 1.80$ \\
Berberis vulgaris & PEG & $-31.83 \pm 4.20$ \\
& 20\% ethanol & $-30.35 \pm 1.80$ \\
& $95 \%$ ethanol & $-4.25 \pm 1.24$ \\
& Water & $-2.02 \pm 3.41$ \\
& PEG & $-20.22 \pm 8.34$ \\
& 20\% ethanol & $-15.87 \pm 4.83$ \\
& $95 \%$ ethanol & $-13.93 \pm 4.70$ \\
\hline
\end{tabular}

Values represent the mean of three replicates \pm standard deviation

Negative sign (-) indicates inhibition, Positive sign (+) indicate activation

\section{Discussion}

\subsection{Acetylcholinesterase Inhibitors}

Acetylcholinesterase inhibitors from Plants Several reviews on the newly discovered AChE inhibitors obtained from plants, fungus have also been published over the last years [9-11]. The majority of these AChE inhibitors belong to the alkaloid group, including indole, isoquinoline,quinolizidine,piperidine and steroidal alkaloids. On the other hand, several non-alkaloidal and potent AChE inhibitors have been obtained from natural sources, including terpenoids, flavonoids and other phenolic compounds.

\subsection{Cholinergic and Anti-cholinergic Effect}

The fractions of Thymus vulgaris, calluna vulgaris and Berberis vulgaris were evaluated for their effect enzyme AChE (E. C. 3.1.1.7). This enzyme is important for breaking acetylcholine (Table 1) which is a substrate of this enzyme that acts on a neurotransmitter in both the peripheral nervous system and central nervous system. Since AD, one of the most common cause of death worldwide, has become a threat to public health, new treatment strategies based on medicinal plants have been focused [8]. In abnormal activation of AChE, acetylcholine will degrade rapidly, especially in the brain and this is associated with Alzheimer's disease (AD). In this study it was found that most of the extracts fraction significantly inhibited the activity of AChE enzyme especially Berberis vulgaris which showed the highest inhibitory effect. These data are in agreement with that [8]. It has been suggested that, the main functional components found in Berberis extract are alkaloids such as berberine which is the main active ingredient of Berberis vulgaris, therefore the compound might have a therapeutic potential for treatment of Alzheimer's disease.

Recently, a study concerning the AChE inhibitory of Thymus vulgaris essential oil was reported. Such results proved the diversity of results depending on the chemical composition. In contrary to this some fractions of, Thymus vulgaris showed stimulatory effect of the enzyme, this effect is in agreement with other researchers who have reported its use in memory improvement that is treatment of hyperactive disorder, therefore the compound might have a therapeutic potential for treatment and other diseases involving AChE hyperactivity, where it was stated [7].

\section{Conclusion}

This study has demonstrated different degrees of AChEI potency for the essential oil extracts, and could be considered as a first level screening stage for these extracts' suggest further tests for therapeutic index and quantal dose relationships to aid clinical decisions and recommendations. Given the complexity of $\mathrm{AD}$, involving many pathological processes, treatment with multifunctional compounds has become an important area of research. Given that the E. Os reported here have desirable multifunctional bioactive properties, further work investigating the benefits of these herbs in reducing the incidence and severity of $\mathrm{AD}$ (and other age related diseases) warrants further attention.

\section{References}

[1] M. Ayaz, Sadiq A, Junaid M, Ullah F, Subhan F, Ahmed J. Neuroprotective and anti-aging potentials of essential oils from aromatic and medicinal plants. Front Aging Neurosci. 2017; 9: 168 .

[2] K. Wollen, A. Alzheimer's disease: the pros and cons of pharmaceutical, nutritional, botanical, and stimulatory therapies, with a discussion of treatment strategies from the perspective of patients and practitioners. Altern. Med. Rev. 2010; 15 (3): 223-24.

[3] D .Ghareeb, Newaairy A, El-rashidy F, Hussein H, Ali A (2010). Efficacy of natural extracts of Ginko biloba and Berberry and synthrtic derivative of genistein as acethylcholinestarase inhibitors, comparative study with Aricept effect. J. Biochem. Biotechnol. 1: 15-20. 
[4] K. Yoo, Hwang IK, Lim BO, Kang TC, Kim D W.et al (2006). Berberry Extract Reduces Neuronal Damage and N-MethylD-aspartate Receptor 1 Immuno reactivity in the Gerbil hippocampus after Transient Fore brain Ischemia. Biol. Pharm. Bull. 29 (4): 623-628.

[5] S. Dandle, Miguel MG, Duarte J, Faleiro ML, Sousa MJ, Lima AS, FigueiredoAC, Barroso JG, Pedro LG (2011).Acetyl cholinesterase inhibition activity of Portuguese Thymus species essential oils. J. Essent. Oil Bear. P1., 14: 140-150.

[6] M. Asai. NI wata, A. Yoshikawa.,Y. Aizaki, S. Ishiura, T. Saido and Maruyama, K. (2007) Berberine alters the processing of Alzheimer's amyloid precursor 152 protein to decrease Abeta secretion. Biochem. Biophys. Res. Commun. 352 (2) 498-502.

[7] P. Williams, Sorribas A, Howes MJ. Natural Products as a source of Alzheimer's drugs leads. Nat. Prod. Rep. . 2011; 28: $48-77$.

[8] G. Ellman .,Courtney K.D., Andres VJr, Featherstone R.M. A new and rapid colorimetric determination of acetylcholinesterase activity. Biochem. Pharmacol. 1961; 7: 88-95.

[9] Houghton PJ, Y Ren, MJ Howes. Acetylcholinesterase inhibitors from plants and fungi. Nat. Prod. Rep. 23 (2006): 181-199. 13.

[10] Williams P, A Sorribas, MJ Howes. Natural Products as a source of Alzheimer's drugs leads. Nat. Prod. Rep. 28 (2011): 48-77. 14

[11] Mukherjee PK, V Kumar, M Mal, PJ Houghton. Acetylcholinesterase inhibitors from plants. Phytomedicine 14 (2007): 289-300. 15.

[12] Orhan G, I Orhan, N Subutay-Oztekin, F Ak, B Sener. Contemporary anticholinesterase pharmaceuticals of natural origin and their synthetic analogues for the treatment of Alzheimer's disease. Recent. Pat. CNS Drug. Discov. 4 (2009): 43-51.

[13] Rounsaville TJ, Ranney TG. Ploidy levels and genome sizes of Berberis L. and Mahonia nutt. species, hybrids, and cultivars. Hortscience. 2010; 45: 1029-33.

[14] Tomosaka H, Chin YW, Salim AA, Keller WJ, Chai H, Kinghorn AD. Antioxidant and cytoprotective compounds from Berberis vulgaris (Barberry) Phytother Res. 2008; 22: 979-81.

[15] Saied S, Begum S. Phytochemical studies of Berberis vulgaris. Chem Nat Compd. 2004; 40: 137-40. 